\title{
3. Erklärungsansätze zum Zusammenhang von Mediennutzung und Schulleistung
}

Im vorherigen Kapitel konnte dargelegt werden, dass zwischen einer exzessiven Mediennutzung und verminderter Schulleistung ein korrelativer Zusammenhang besteht. Offen geblieben ist, wie genau sich dabei die Mediennutzung auswirkt. Zum Wirkzusammenhang von Mediennutzung und schulischer Leistung gibt es unterschiedliche Annahmen. Überblicke zu den verschiedenen Wirkhypothesen finden sich beispielsweise bei Morgan und Gross (1982), Beentjes und Van der Voort (1988), Anderson, D. R., Huston, Schmitt, Linebarger und Wright (2001) sowie bei Shin (2004). Die Hypothesen zum Zusammenhang zwischen Mediennutzung von Kindern und deren Schulerfolg lassen sich in zwei große Kategorien einteilen: „Stimulierungshypothesen“ und „Minderungshypothesen“ (Shin, 2004). Stimulierungshypothesen gehen beispielsweise im Bereich des Fernsehens davon aus, dass gut gestaltete Kinderprogramme Schulleistungen von Kindern verbessern, weil die Kinder aus den Programmen lernen können. Für die Computerspiele werden als lernförderliche Wirkungen z. B. verbesserte visuelle Aufmerksamkeit (Castel, Pratt \& Drummond, 2005), visuelle Wahrnehmungsleistung (Subrahmanyam \& Greenfield, 1994), mentale Rotation (De Lisi, 2002) und computerspielspezifische Heurismen und Gewinnstrategien (Kraam-Aulenbach, 2000) diskutiert.

Minderungshypothesen auf der anderen Seite stützen sich auf die Annahme, dass Mediennutzung entweder die intellektuellen Fähigkeiten des Betrachters direkt vermindern oder zu spezifischem Verhalten führen kann, welches den Schulerfolg von Kindern beeinträchtigt (Shin, 2004). Aufgrund der oben referierten Befundlage sowie des speziellen Erkenntnisinteresses den negativen Zusammenhang zwischen Mediennutzung und Schulleistung aufzuklären, kommt den Minderungshypothesen eine besondere Bedeutung zu. Im Folgenden sollen drei Erklärungsansätze aus dem Bereich der Minderungshypothesen dargestellt werden, die auch immer wieder durch unsere eigenen Daten nahe gelegt werden:

1. Der Gebrauch von Medien kann ab einem gewissen Grad Lernaktivitäten, Freizeitverhalten und Erholungszeiten zeitlich verdrängen und dadurch zu einer verminderten Schulleistung führen. Des Weiteren drohen bei exzessiver Nutzung suchtähnliche Nutzungsmuster, die sich besonders nachhaltig auf die schulische Leistungsfähigkeit auswirken können. Die intensivierte Mediennutzung führt darüber hinaus zu verminderten Schlaf- und Erholungszeiten. Diese Annahmen werden im Rahmen der Zeitverdrängungshypothese diskutiert.

2. Die hohe Präferenz gewalthaltiger Medienangebote kann zu einer verstärkten Aggressivität und Desensibilisierung für reale Gewalt der Nutzer führen, und 
damit die Partizipation an schulischen Lernprozessen beeinträchtigen. Diese Annahmen werden im Rahmen der Inhaltshypothese diskutiert.

3. Die hohe Präferenz gewalthaltiger Medienangebote könnte neueren Daten zufolge zudem dafür verantwortlich sein, dass schulische Lernprozesse unmittelbar beeinträchtigt werden. Diese Annahme wird im Rahmen der Löschungshypothese diskutiert.

\subsection{Zeitverdrängungshypothese}

Diese Hypothese geht von der Annahme aus, dass die Beschäftigung mit Medien an die Stelle anderer nichtmedialer Freizeit- sowie von Lernaktivitäten tritt, diese verdrängt und damit zu einer Einengung des Freizeiterlebens führt. Dies ist nicht ohne Belang für schulische Leistungen. So bietet gerade ein vielseitiges Freizeitverhalten die Chance für wichtige Lernerfahrungen, den Erwerb sozialer Kompetenzen und körperlicher sowie psychischer Gesundheit und kann damit als wichtige Quelle schulischer und beruflicher Erfolge gelten (Anderson, D. R. et al., 2001). Zudem führt eine intensive Mediennutzung auch zu einer direkten Reduktion insbesondere intellektuell herausfordernder Tätigkeiten wie z. B. Hausaufgaben machen oder Lernen (vgl. Beentjes \& Van der Voort, 1989; Harrison \& Williams, 1986; Shin, 2004; Valkenburg \& Van der Voort, 1994) und verringert dadurch letztendlich die schulischen Leistungen (Koshal, Koshal \& Gupta, 1996). So bemerken Gentile et al. (2004) einfach wie treffend, dass bei einer durchschnittlichen wöchentlichen Computerspielzeit von sieben Stunden in diesen sieben Stunden nun nicht mehr gelesen, Hausaufgaben gemacht und kreativen Beschäftigungen nachgegangen werden könne.

Solange die Nutzung der Mediengeräte in einem gewissen Rahmen bleibt, besteht kaum Grund zur Beunruhigung. Vielfach ufert der Konsum jedoch aus, wobei auf die Computerspiele ein besonderes Augenmerk gelegt werden muss. Untersuchungen zufolge neigt ein gewisser Anteil aller computerspielenden Kinder und Jugendlichen zu einem „exzessiven“ Computerspielverhalten (je nach zugrunde gelegter Definition ca. 3 bis 13 Prozent (vgl. Feierabend \& Klingler, 2003b; Funk, 1993; Roberts et al., 2005; van Schie \& Wiegman, 1997)), dem eine hohe Relevanz für schulleistungsbezogene Parameter zukommen kann:

„Eine ausufernde Flucht in die Virtualität kann dazu führen, dass eine Person oder eine ganze Gesellschaft den Erfordernissen des Alltags nicht mehr nachkommt. Das reale Leben läuft dann Gefahr, nur noch dazu zu dienen das virtuelle Leben zu ,unterhalten"“ (te Wildt, 2004, S. 576).

Wenn auch nicht bei moderatem Spielen, so leidet bei stark erhöhtem Computerspielverhalten das Lernen für die Schule. Gentile et al. (2004) kommen in einer Studie mit einer Stichprobe von Acht- und Neuntklässlern $(n=607)$ zu dem Ergebnis, dass die Spieldauer auch dann signifikant mit schlechten Noten korreliert, wenn die Nutzung von Gewaltinhalten statistisch kontrolliert wird. Eine besondere Verschär- 
fung des zeitlichen Engpasses ergibt sich zudem dadurch, dass mit erhöhter Computerspielnutzung auch weitere Medien ausgiebiger genutzt werden. So kommen in einer US-amerikanischen Studie Nichtspieler auf eine tägliche Nutzungszeit übriger Medien von 6 Stunden und 45 Minuten, Normalspieler (5 Minuten bis 1 Stunde Spielzeit pro Tag) auf 8 Stunden und 52 Minuten und Intensivspieler (mehr als 1 Stunde Spielzeit pro Tag) auf 11 Stunden und 53 Minuten (Roberts et al., 2005).

\section{Computerspielsucht}

Ungeachtet dieser hohen Nutzungszeiten ist in der Forschung sowie klinischen Praxis nach wie vor umstritten, ob und ggf. unter welchen Umständen Computerspiele süchtig machen können (vgl. te Wildt, 2004). Ein dem Computerspielen zugrunde liegendes Suchtgeschehnis erscheint zunächst aufgrund der hohen Nutzungszeiten vieler Spieler und ihrer selbstberichteten Unfähigkeit, das Spielverhalten zu reduzieren, plausibel. Gerade Vielspieler empfinden sich selbst, insbesondere im Anschluss an exzessive Spielphasen, als computerspielsüchtig und berichten von gescheiterten Versuchen, die Spielzeit zu reduzieren. Betroffene Eltern wissen mit der bestehenden Situation oftmals kaum umzugehen.

Eine Ursache für die intensiven Nutzungszeiten wird in der besonderen Attraktivität des interaktiven Mediums gesehen, denn Computerspiele haben einen ganz besonderen Reiz für den Nutzer: Das Medienerlebnis vollzieht sich hier in unmittelbarer Abhängigkeit von den Handlungen des Spielers, der aktiv die Spielnarration vorantreibt (vgl. Klimmt, 2004). Die Motivations- und Anreizfunktionen für ein Aufrechterhalten des Spielerlebens sind entsprechend vielfältig: Als besondere Spielmotive werden z. B. das Erleben von Herausforderungen und Wettbewerb, die Ausübung von Macht und Kontrolle (Selbstwirksamkeit), die Entwicklung und Erprobung von Identitätsentwürfen, das Erleben von Geselligkeit, die Flucht aus dem Alltag (Eskapismus), Stressabbau und das Erleben eines emotionalen Verschmelzungszustandes mit dem Spiel im Sinne eines FLOW-Erlebens diskutiert (Kunczik \& Zipfel, 2004). Von entscheidender Bedeutung ist insbesondere die zeitliche Offenheit der Nutzung. Begrenzt sich Fernsehen häufig zumindest noch auf den Erwerb oder die gezielte Auswahl attraktiver Filme und Sendungen, können favorisierte Computerspiele tage-, wochen- oder auch monatelang gespielt werden, ohne dass das Rezeptionsgeschehen wie bei Filmmedien ein natürliches Ende erreichen würde ${ }^{6}$.

Doch kann man von Computerspielen auch süchtig werden, ähnlich wie von Alkohol oder Zigaretten? Die Forschungslage spricht dafür, dass eine intensive und anhaltende Nutzung von Computerspielen physiologisch einige Parallelen zu stoffgebunden Süchten aufweist. So wird befürchtet, dass sich bei chronisch intensiver

6 Die gilt insbesondere für Multiplayerspiele, jedoch auch eingeschränkt für Singleplayerspiele, die häufig ein mehrmaliges Durchspielen mit unterschiedlichen nichtlinearen Handlungsverläufen erlauben. 
Spielnutzung aufgrund der exzessiv belohnenden Wirkungen des Spiels im Sinne einer Verhaltenssucht ${ }^{7}$ psychotrope Effekte ${ }^{8}$ über körpereigene, biochemische Veränderungen einstellen, wie sie auch bei stoffgebundenen Süchten zu beobachten sind (Grüsser \& Rosemeier, 2004; Holden, 2001).

\section{Kasuistik eines jungen Mannes mit einer Abhängigkeit von einem Internet-Rollenspiel (von Bert T. te Wildt)}

Ein junger Mann zog sich aufgrund persönlicher Niederlagen zunehmend aus seiner konkret-realen Umwelt zurück, um sich gleichzeitig immer mehr in der virtuellen Welt eines Rollenspiels im Internet zu verstricken. Nach einem zunächst viel versprechenden Beginn seiner schulischen Karriere hatten die schulischen Leistungen des damals 16-jährigen in der Oberstufe des Gymnasiums schlagartig nachgelassen, als die Ehe der Eltern in eine Krise geriet. Bald nach der Scheidung der Eltern brach der Junge wegen einer drohenden Nicht-Versetzung gegen den Willen der Eltern die 11. Klasse ab, ohne für sich eine Perspektive für einen Ausbildungsplatz zu entwickeln. In der Folge zog er sich immer mehr in sein Zimmer zurück, um dort Computerspiele zu spielen. Wie seine Freunde hatte er schon als Kind häufig Konsolenspiele gespielt, wobei er in seiner Freizeit mit diesen jedoch immer auch aktiv Sport betrieben hatte. Nach seinem Schulabbruch verlor er den Kontakt zu den Freunden fast vollständig.

In dem Rollenspiel World of Warcraft, in dem er sich nun täglich bis zu 12 Stunden aufhielt, spielte er den Helden, der er in der konkret-realen Welt bisher nicht sein konnte. Mit Clans verabredete er sich täglich dazu, Drachen zu töten, Burgen zu erobern und andere Clans zu bekämpfen. Irgendwann verließ er sein Zimmer nur noch, um ins Bad zu gehen oder sich Essen aus der Küche zu holen, welches er dann vor seinem Computer einnahm. Über einen Zeitraum von zwei Jahren exzessiven Computerspielens baute er physisch und seelisch immer mehr ab. Saß er nicht vor seinem Computer, konnte er despotisch und reizbar werden, sodass immer wieder Streit mit der Mutter entstand, insbesondere dann, wenn sie versuchte, inn dazu zu animieren, sich einen Ausbildungsplatz zu suchen. Jegliche Hilfsangebote wurden von dem mittlerweile 18-jährigen ausgeschlagen. Da gegen seinen Willen keine Behandlung möglich war, sah sich die Mutter schließlich dazu gezwungen, den Internet-Anschluss zu kündigen. In Folge dessen reagierte der Sohn verzweifelt und höchst aggressiv, sodass der sozialpsychiatrische Dienst notfallmäßig erneut vorbeikommen musste. Mit der klaren Haltung der Mutter, den Sohn vor die Tür zu setzen, sollte er sich nicht behandeln lassen, gelang es schließlich, ihn zu einer stationären Behandlung zu bewegen.

Die Befürworter dieser These argumentieren, dass das menschliche Gehirn eine Belohnung immer gleich behandelt, unabhängig davon, ob diese durch einen bestimmten Stoff (z. B. Alkohol) oder eine bestimmte Verhaltensweise (z. B. Glücksspiel) induziert wird (Holden, 2001). In Einklang mit diesen Befunden konnte festgestellt

7 Eine anerkannte Verhaltenssucht ist beispielsweise das pathologische Glücksspiel, diskutiert werden auch Arbeitssucht, Sexsucht und Sportsucht (Grüsser, S. M. \& Thalemann, C. N., 2006; Poppelreuter \& Gross, 2000)

8 Psychoaktive Veränderung der Psyche und des Bewusstseins. Eine solche Änderung kann sehr subtil sein, z. B. eine leichte Anregung, Entspannung oder Stimmungsänderung, oder auch tiefreichender im Sinne eines stark veränderten Bewusstseinszustands oder sog. Trips, der aber nur durch starke Halluzinogene ausgelöst werden können. 
werden, dass Computerspielen mit einer hohen Dopaminausschüttung einhergeht (Koepp et al., 1998). Dem dopaminergen mesolimbischen Belohnungssystem wird eine entscheidende Bedeutung für die Entstehung und Aufrechterhaltung von Suchterkrankungen zugesprochen.

Welche hirnphysiologischen Parallelen zwischen exzessivem Computerspielen und stoffgebundenen Suchterkrankungen bestehen, konnte zudem kürzlich eine Studie der Charité Universitätsmedizin Berlin eindrucksvoll aufzeigen. In der klinischexperimentellen Studie wurden Probanden mit unterschiedlichen neutralen und suchtsassoziierten Bildreizen konfrontiert und ihre Hirnaktivitäten parallel mittels Elektroenzephalogramm (EEG) und Elektromyogramm (EMG) aufgezeichnet. Es zeigte sich, dass Alkoholiker hirnphysiologisch in sehr ähnlicher Weise auf die gezeigten Alkoholbilder reagieren wie Computerspielsüchtige auf die präsentierten Computerspielbilder. Als besonders auffällig interpretiert die Forschergruppe den Befund, dass durch die jeweils suchtassoziierten Reize nicht nur eine besonders starke Erregung ausgelöst wird (EEG), sondern die Daten zum Startle-Reflex (Schreckreflex, EMG) gleichzeitig auf eine besonders positive Bewertung des jeweils relevanten Suchtstimulus hindeuten. Computerspielbilder wirken auf Exzessivspieler demnach neurobiologisch in ganz ähnlicher Weise wie Bilder von Alkohol auf Alkoholiker. Eine erhöhte Aufmerksamkeit für die suchtbezogenen Reize zeigte sich für stoffgebundene Süchte und Verhaltenssüchte nahezu identisch (Thalemann \& Grüsser, 2005).

Trotz der vielfältigen Hinweise, welche die Annahme einer Computerspielsucht rechtfertigen, gilt diese bislang nicht als klinisch anerkannte Suchtstörung. Als Verhaltenssucht ist bislang nur das Pathologische Glücksspiel in die Nosologie eingegangen, findet sich hierbei jedoch im DSM-IV (Saß, Wittchen, Zaudig \& Houben, 2003) unter der Rubrik ,Abnorme Gewohnheiten und Störungen der Impulskontrolle" wieder. Exzessives Computerspielen wird daher als Sonderform einer substanzungebundenen Suchterkrankung mit auffälligen Parallelen zu anderen Verhaltenssuchtformen (Thalemann \& Grüsser, 2005), aber auch als Störung der Impulskontrolle, die Ausdruck anderer zugrunde liegender Erkrankungen sein könnte (te Wildt, 2004) diskutiert. Auffallend ist die Begriffsverwirrung innerhalb der Debatte, indem Termini wie Computerspielsucht, exzessives Computerspielverhalten, exzessive Computernutzung, Internetsucht, Online-Sucht, problematischer Internetgebrauch und pathologischer Computergebrauch z. T. synonym oder aber in Abgrenzung vermeintlich spezifischer Mediensüchte gebraucht werden. Eine einheitliche Nomenklatur ist bislang ausgeblieben (te Wildt, 2004), was auf die Vielfalt und Veränderlichkeit der neuen digitalen Medien (Wartella, Lee \& Caplovitz, 2002) und ihren zunehmenden Konvergenzcharakter ${ }^{9}$ zurückgeführt werden kann.

9 Medienkonvergenz beschreibt die Verschmelzung unterschiedlicher bislang separierter Medienfunktionen in einem einzigen neuen Medium. Neue digitale Medien sind typischerweise Konvergenzmedien, z. B. der Computer, mit dem Filme geschaut und Spiele gespielt werden können, aber auch im Internet gesurft werden kann. 
Aus diesem Grunde werden bislang je nach Studie unterschiedliche Maßstäbe an Computerspielsucht herangetragen. Als einheitliches Primärkennzeichen gilt eine hohe Nutzungshäufigkeit und eine insgesamt hohe Nutzungsdauer bestehend über einen längeren Zeitraum von mehreren Wochen oder Monaten. Von Bedeutung ist darüber hinaus das Vorliegen einer inadäquaten Stressregulationswirkung des Computerspielens. Schon seit Mitte der 1980er Jahre wird darauf verwiesen, dass Computerspiele häufig in Anspannungssituationen gespielt werden und sich Spieler im Anschluss als entspannter beschreiben als vor dem Spielen (Kestenbaum \& Weinstein, 1985). Dies könnte eine für Suchterkrankungen typische, stressregulative Funktion des Mediums zum Ausdruck bringen wie sie von Grüsser und Rosemeier (2004) bei Ihrer Definition von Computerspielsucht in den Vordergrund gestellt wird. Die ursprüngliche Funktion des Spielens (Unterhaltung) tritt zunehmend hinter einer dysfunktionalen Wirkung (Stressregulierung) zurück.

Ähnliches beschreiben der Erziehungswissenschafter Bergmann und der Neurobiologe Hüther (2006) in ihrem Buch „Computersüchtig. Kinder im Sog der Medien“. Auch für sie ist von ausschlaggebender Bedeutung, wie Computerspiele eingesetzt werden. Starke und glückliche Kinder, die ihren Bedürfnissen auf vielfältige Weise nachkommen, seien kaum anfällig für Computerspielsucht. Die Autoren sehen jedoch dann eine besondere Suchtgefahr gegeben, wenn Computerspiele schon in jungen Jahren als primäres Mittel eingesetzt werden, um Glücksgefühle zu erlangen und negative Gefühle zu überwinden. Diese Belohnungsmechanismen werden im Gehirn mit der Zeit immer stärker verankert und die Gefahr einer Abhängigkeit nimmt zu.

Grüsser und Thalemann (2006) beschreiben in ihrem Ratgeberbuch „Computerspielsüchtig? Rat und Hilfe“ Indikatoren, die auf eine Computerspielsucht hindeuten, wie:

- Einengung des Verhaltensmusters (Computerspielen wird zur wichtigsten Aktivität, dominiert Denken, Fühlen und Verhalten)

- Regulation von negativen Gefühlszuständen (Affekten)

- Zunehmende Steigerung der Nutzungsdauer (Toleranzentwicklung)

- Entzugserscheinungen (z. B. Nervosität, Unruhe, Zittern, Schwitzen...)

- Kontrollverlust

- Rückfälle

- Schädliche soziale und berufliche/schulische Konsequenzen (aus Grüsser \& Thalemann, 2006, S. 29)

Es besteht jedoch nach wie vor eine große Uneinigkeit darüber, in welcher Weise die Kriterien untereinander gewichtet werden und wie sie in geeigneter Weise zu erfassen sind. Aufgrund der Heterogenität in Operationalisierung und Messung fallen auch die bislang vorliegenden Prävalenzschätzungen uneinheitlich aus. Eine Interpretation dieser Schätzungen wird dadurch erschwert, dass in den verschiedenen Untersuchungen hochgradig divergente Untersuchungspopulationen untersucht wurden, die sich in Alter und Computerspielnutzungsstatus z. T. deutlich voneinander 
unterscheiden. Trotz dieser Mängel erlauben die Daten jedoch eine erste Einschätzung über die Verbreitung von „Computerspielsucht“ unter Kindern und Jugendlichen.

In einer koreanischen Studie von Yang (2001) wurden insgesamt 1.296 Schüler und Schülerinnen 7. bis 12. Schulklassen zu ihrer Computernutzung befragt. Zum Einsatz kam eine Internetsuchtskala, das Computer-Related Addictive Behavior Inventory (CRABI), welche von den Autoren für die Erfassung von Problemen in $\mathrm{Zu}$ sammenhang mit Computernutzung adaptiert wurde. Die Autoren ermittelten hierbei einen Anteil von 6,1 Prozent aller Kinder und Jugendlichen, die eine aktuelle Computersucht aufweisen.

In einer Studie von Whang (2003) wurden 13.588 Teilnehmer anhand der modifizierten Form einer Internetsucht-Skala eingestuft. 3,5 Prozent wurden als süchtige Nutzer, 18,4 Prozent als suchtgefährdete Nutzer diagnostiziert.

Griffiths, Davies \& Chappell (2004) vergleichen in ihrer Untersuchung jugendliches und erwachsenes Computerspielverhalten miteinander. Hierzu verwendeten sie einen Online-Fragebogen, der von Spielern des Online Spiels Everquest ausgefüllt wurde. Untersucht wurde hierbei also eine Unterpopulation von Spielern eines bestimmten Online-Spiels. Den Ergebnissen zufolge waren jugendliche Spieler eher männlich und tendierten stärker dazu, die Schule oder den Beruf für das Computerspielen zu opfern. Insgesamt konnte eine Quote von 9 Prozent computerspielsüchtiger Jugendlicher innerhalb dieser Nutzergruppe ermittelt werden.

Johansson und Götestam (2004) untersuchte eine norwegische Stichprobe von 3.237 Jugendlichen im Alter von 12 - 18 Jahren. 63,6 Prozent der Jugendlichen wurden als regelmäßige (wöchentliche) Computerspieler und 36,7 Prozent als Gelegenheitsspieler eingestuft. Zusätzlich wurde zur Suchtklassifikation das Diagnostic Questionaire for Internet Addiction von Young (1998) eingesetzt. 9,8 Prozent der Jugendlichen erfüllten die Kriterien für eine Suchtgefährdung (14,5\% der Jungen und $5 \%$ der Mädchen). Als pathologisch süchtige Computerspieler wurden 2,7 Prozent der Jugendlichen eingestuft (4,2 \% der Jungen und 1,1 \% der Mädchen).

In einer aktuellen Querschnittsuntersuchung von Grüsser, Thalemann, Albrecht \& Thalemann (2005) wurden 323 Kinder sechster Schulklassen (148 Mädchen, 175 Jungen) hinsichtlich ihrer Computerspielnutzung befragt. Zum Einsatz kam dabei der Fragebogen zum Computerspielverhalten bei Kindern (CSVK), der sich an bestehende Suchtkriterien des DSM-IV (pathologisches Glücksspiel) und ICD-10 (Abhängigkeitserkrankung) anlehnt. Dabei wurden Dimensionen wie Dauer und Häufigkeit der Nutzung, inadäquate Funktionen (Vergessen von Ärger) sowie negative soziale Folgen des Spielens (z. B. Streit mit der Familie) und die gedankliche Beschäftigung mit dem Spielen einbezogen. Ab einem kritischen Cut-Off Wert, der eine überdurchschnittliche Zustimmung zu den Unterdimensionen zum Ausdruck bringt, wurden die betreffenden Kinder als exzessiv spielend bzw. computerspielsüchtig eingestuft. Hierbei kam die Forschergruppe zu dem Ergebnis, dass insgesamt 30 der 323 Kinder $(9,3 \%$, hiervon 7 Mädchen und 23 Jungen) die Kriterien für Computerspielsucht erfüllten. 
Damit liegen aktuelle Prävalenzschätzungen für Computerspielsucht zwischen 3 und 9 Prozent, je nach Untersuchungspopulation und eingesetzter Messinstrumente (einen Überblick gibt Tabelle 1).

Tabelle 1: Studien zur Prävalenz von Computerspielsucht

\begin{tabular}{|c|c|c|c|}
\hline Autor (Jahr) & Stichprobe & Instrument & $\begin{array}{l}\text { Prävalenzrate } \\
\text { (Gefährdung) }\end{array}$ \\
\hline Yang (2001) & $\begin{array}{c}\mathrm{N}=1.296 \text { Jugendliche } \\
\mathrm{w}=641 ; \mathrm{m}=655 \\
14,8 \text { Jahre } \\
\text { 7.- } 12 . \text { Klassen }\end{array}$ & $\begin{array}{l}\text { Computer-Related Addictive } \\
\text { Behavior Inventory (CRABI) }\end{array}$ & $\begin{array}{c}6,1 \% \\
(X)\end{array}$ \\
\hline Whang (2003) & $\begin{array}{l}N=13.588 \text { Personen } \\
w=5.710 ; m=7.878\end{array}$ & $\begin{array}{l}\text { Internet Addiction Scale } \\
\text { (modifiziert) }\end{array}$ & $\begin{array}{c}3,5 \% \\
(18,4 \%)\end{array}$ \\
\hline $\begin{array}{l}\text { Griffiths, Davies \& } \\
\text { Chappell (2004) }\end{array}$ & $N=540$ & Online Fragebogen & $\begin{array}{l}9 \% \\
(X)\end{array}$ \\
\hline $\begin{array}{l}\text { Johansson \& Gö- } \\
\text { testam (2004) }\end{array}$ & $\begin{array}{c}N=3.237 \\
12-18 \text { Jahre }\end{array}$ & $\begin{array}{l}\text { Diagnostic Questionaire for } \\
\text { Internet Addiction (modifi- } \\
\text { ziert) }\end{array}$ & $\begin{array}{c}2,7 \% \\
(9,8 \%)\end{array}$ \\
\hline $\begin{array}{l}\text { Grüsser, Thale- } \\
\text { mann, Albrecht \& } \\
\text { Thalemann (2005) }\end{array}$ & $\begin{array}{c}N=323 \\
w=148 ; m=175 \\
M W=11,83 \text { Jahre }\end{array}$ & $\begin{array}{l}\text { Fragebogen zum Compu- } \\
\text { terspielverhalten bei Kindern } \\
\text { (CSVK) }\end{array}$ & $\begin{array}{c}9,3 \% \\
(X)\end{array}$ \\
\hline
\end{tabular}

Die Datenlage bietet somit einen deutlichen Hinweis darauf, dass einer exzessiven Computerspielnutzung tatsächlich ein klinisch relevantes Suchtgeschehen zugrunde liegt. Dies könnte von besonderer Tragweite sein, denn hier drohen weitere Risiken für die betroffenen Kinder und Jugendlichen. So weist die Forschungslage schon jetzt darauf hin, dass exzessiv spielende Jugendliche vermehrt zu sozialer Isolation und Konflikten mit Familienangehörigen neigen und zudem verstärkt psychiatrisch/klinische Auffälligkeiten aufweisen wie eine erhöhte Depressivität und Angstneigung, ein geringeres Selbstvertrauen und erhöhte Zwanghaftigkeit (Whang, 2003; Yang, 2001). Yang fasst die Daten seiner Untersuchung wie folgt zusammen: ,Although computer addiction has not been recognized as an official diagnosis, it can be viewed as a problematic behaviour requiring more attention, if it causes problems in daily life and interferes with healthy development during his/her adolescence years." (Yang, 2001, S. 221).

\section{Schlaf- und Erholungszeiten}

Eine weitere relevante Veränderung im Freizeitverhalten bedingt durch exzessive Mediennutzung betrifft reduzierte Schlaf- und Erholungszeiten. Erhöhte Mediennutzungszeiten bei gleich bleibenden oder ebenfalls erhöhten sonstigen nichtmedialen Freizeitaktivitäten können zu der Notwendigkeit führen, den Freizeitanteil im Tagesverlauf zu vergrößern, indem erst später zu Bett gegangen wird (Gillespie, 2002; Van den Bulk, 2004). In einer Befragung von Onlinerollenspielern (Griffiths et al., 
2004) geben 12,5 Prozent der Jugendlichen und 20,8 Prozent der Erwachsenen an, aufgrund des Spielens weniger zu schlafen. Tatsächlich zeigen sich bei Mustern exzessiver Computerspielnutzung Anzeichen für Schlafentzug bei den Spielern (Gillespie, 2002; Tazawa \& Okada, 2001), der mit einem problematischerem Sozialverhalten, verringerter Konzentrationsfähigkeit und verminderter psychischer Gesundheit in Zusammenhang stehen könnte.

In einer aktuellen deutschen Studie (Crönlein et al., 2007) mit im Mittel 13Jährigen $(n=137)$ konnte ein Zusammenhang zwischen dem Fernsehkonsum der Jugendlichen und Schlafstörungen festgestellt werden. Neben der Quantität der Mediennutzung kommt dabei auch dem konsumierten Inhalt eine entscheidende Bedeutung zu. So hängen (vor allem bei Jungen) längere Fernsehzeiten insgesamt mit verkürzten Schlafzeiten zusammen, der häufige Konsum von Gruselfilmen jedoch darüber hinaus (für beide Geschlechter) mit einem gestörten Einschlafen. Die Autoren selbst betonen jedoch den explorativen Charakter dieser Studie, da zum einen nur Haupt- und Realschüler befragt wurden und zum anderen weder die ethnische Zugehörigkeit noch das Bildungsniveau als wichtige Einflussfaktoren auf die Medienzeiten berücksichtigt wurden.

Ähnliche Ergebnisse berichten Heins et al. (2007) auch für Grundschüler. In ihrer Querschnittsbefragung zu Handynutzungsgewohnheiten von 1.933 Schülern (im Mittel 10-jährig) hing eine Schlafenszeit nach 21 Uhr mit einem höheren Alter, männlichem Geschlecht, älteren Geschwistern, der Schullokalisation in der Stadt, dem Besitz eines eigenen Handys und einem Fernseh- und Computerkonsum von mehr als 3 Stunden täglich zusammen. Auch für diese Studie gelten jedoch die oben genannten Einschränkungen.

Gemeinhin handelt es sich beim Schlafen um einen allgemeinen Erholungszustand, der die Regeneration von Körper und Gehirn fördert. Zusätzlich werden im Schlaf insbesondere Gedächtnisinhalte konsolidiert und für weitere Abrufe stabilisiert (vgl. Fenn, Nusbaum \& Margoliash, 2003; Graves, Pack \& Abel, 2001; Payne \& Nadel, 2004; Plihal \& Born, 1997; Siegel, 2001). Lange Zeit galt insbesondere der REMSchlaf („,Rapid Eye Movement“; hier findet sich der höchste Grad an Muskelerschlaffung) als relevant für die Gedächtniskonsolidierung. Diese Hypothese wurde in jüngster Zeit jedoch immer mehr kritisiert (vgl. Vertes \& Eastman, 2000): Neben dem REM-Schlaf scheint auch der Non-REM-Schlaf einen Einfluss auf die Gedächtnisbildung zu haben (vgl. Gais \& Born, 2004). Einige Untersuchungen konnten zeigen, dass nicht nur bei chronischem (weniger als sechs Stunden Schlaf pro Nacht) und totalem Schlafentzug über einen gewissen Zeitraum (vgl. Pilcher \& Huffcutt, 1996; Van Dongen, Maislin, Mullington \& Dinges, 2003), sondern auch bei moderatem vorübergehendem Schlafentzug (24 bis 28 Stunden ohne Schlaf) (Thomas et al., 2000; Williamson \& Feyer, 2000) Beeinträchtigungen in der kognitiven Leistungsfähigkeit zu beobachten sind. Dabei zeigt sich, dass diese Beeinträchtigungen den Betroffenen nicht bewusst sein müssen (vgl. Van Dongen et al., 2003). Es ist somit zu befürchten, dass aus einem erhöhten Fernseh- und Spielverhalten insbesondere in den Abend- und Nachtstunden eine Verringerung der Schlafzeit resultiert, die als 
unmittelbare Konsequenz eine Verminderung schulischer Leistungsfähigkeit nach sich zieht.

\section{Bewegungsarmut und Übergewicht}

Ein letzter wichtiger im Rahmen der Zeitverdrängungshypothese zu behandelnder Punkt ist die durch eine intensive Mediennutzung hervorgerufene Verdrängung der Bewegungsaktivitäten von Kindern und Jugendlichen. Eine Metaanalyse von Marshall, Biddle, Gorely, Cameron und Murdey (2004) berichtet negative Zusammenhänge von Mediennutzung und körperlicher Betätigung sowohl für das Fernsehen $(r=-.096)$ als auch für das Computerspielen $(r=-.104)$. Besonders bei Intensivspielern wird eine ungünstige Energiebilanz, eine Erhöhung des Körperfettanteils und damit langfristig Übergewicht befürchtet (Cordes \& Miller, 2001). In einer Schweizer Untersuchungspopulation von Grundschülern der ersten bis dritten Klasse $(n=872)$ wurde ein eindeutiger Zusammenhang zwischen dem Spielen von elektronischen Spielen und dem BMI ${ }^{10}$ gefunden. Das Risiko der untersuchten Erst- bis Drittklässler für Übergewicht steigt mit jeder zusätzlichen täglichen Computerspielstunde auf das Doppelte an (Stettler, Signer \& Suter, 2004).

Epidemiologische Untersuchungen zeigen im Allgemeinen eine zunehmende Bewegungsarmut bei Kindern und Jugendlichen und eine Abnahme der körperlichen Fitness. Im ersten deutschen Kinder- und Jugendsportbericht wird ausgeführt, dass im Jahre 2000 gegenüber 1975 eine durchschnittliche physiologische Leistungsabnahme von 10 Prozent feststellbar ist, mit den größten Unterschieden im Bereich der Grundlagenausdauer und Beweglichkeit (Schmidt, Hartmann-Tews \& Brettschneider, 2003). Aus vielfältigen Gründen ist Bewegung und körperliche Betätigung für schulische Leistungen von Bedeutung. Neurobiologischer Forschung zufolge steht Bewegung mit Intelligenzentwicklung in direktem Zusammenhang. So spielt Sport eine wichtige Rolle für die Hirndurchblutung und die Vernetzung der Hirnzellen untereinander. Gerade im frühen Lebensalter wird die neuronale Plastizität am stärksten über Bewegung beeinflusst (Kubesch, 2002). Bewegung hat einen stimulierenden Einfluss auf die hippocampale Neurogenese und fördert Lernprozesse und die Anpassungsfähigkeit des Gehirns (Kubesch, 2002). So kommt auch eine Längsschnittstudie zur Bewegung im Grundschulalter zu dem Resultat, dass sich eine erhöhte sportliche Aktivität positiv auf die soziale Entwicklung, Konzentrationsfähigkeit und Lernfreude auswirkt (Müller \& Petzold, 2002). Myrtek und Scharff (2000), die die Auswirkungen der Mediennutzung insbesondere auf körperliche und emotionale Befindlichkeit untersucht haben, kommen ferner zu dem Ergebnis, dass die körperliche Aktivität der Vielseher eingeschränkt ist und dass sie weniger soziale Kontakte pflegen. Fernseh-Vielnutzer können weniger gut mit Stress umgehen und bringen durch den als stark empfundenen Schulstress auch schlechtere Noten nach 
Hause (Myrtek \& Scharff, 2000). Darüber hinaus konnte die Freiburger Forschungsgruppe durch eine genaue Messung des Kommunikationsverhaltens ermitteln, dass Vielseher auch in ihren kommunikativen Fähigkeiten eingeschränkt sind. Eine entwickelte kommunikative Kompetenz ist vor allem für die Leistungen im Fach Deutsch von enormer Bedeutung (Myrtek \& Scharff, 2000).

Zusammengefasst können in Bezug auf die Zeitverdrängungshypothese die folgenden Punkte festgehalten werden: Eine zeitlich ausufernde Nutzung von Medien kann zu einem eingeengten Freizeitverhalten führen und damit die Partizipation an alltäglichen Lernprozessen und sozialen Kommunikationserfahrungen vermindern. Weiterhin besteht das Risiko einer zeitlichen Verdrängung von außerschulischen Lernaktivitäten wie die Erledigung von Hausaufgaben sowie nachmittägliche Vertiefung des Unterrichtsstoffes. Darüber hinaus werden klinische Folgen einer intensiven Mediennutzung diskutiert. Neben Schlafentzug, Bewegungsarmut und Übergewicht ist ein Suchtpotential der Medien, insbesondere der Computerspiele, von zunehmender gesellschaftlicher Relevanz. Für all diese Wirkprozesse liegen berechtigte Annahmen darüber vor, dass über sie schulische Leistungen unmittelbar herabgesetzt werden können.

\subsection{Inhaltshypothese}

Lag der Fokus im vorherigen Kapitel auf den reinen Mediennutzungszeiten sowie deren Folgen für die schulische Leistungsentwicklung, sollen in diesem Abschnitt Forschungsdaten vorgestellt werden, die sich im Sinne der Minderungshypothesen auf die Wirkung der genutzten Inhalte konzentrieren. Eine bedeutsame Rolle kommt dabei dem Konsum gewalthaltiger Medienangebote zu. So kann der Konsum gewalthaltiger Medieninhalte mittels seiner negativen Wirkung auf Aggressivität und aggressives Verhalten auch negative Effekte auf den Schulerfolg haben (Anderson, D. R. et al., 2001; Huesmann, 1986). Dieser Argumentation folgend, sollen zunächst Befunde zum Zusammenhang von Mediengewalt und Aggressivität bzw. aggressivem Verhalten vorgestellt werden.

\subsubsection{Mediengewalt und Aggressivität}

„There is little doubt that viewing television violence has a causal effect on aggressive behavior, though there is disagreement about the magnitude and social importance of the effect." (Huston \& Wright, 1998, S. 1043).

$\mathrm{Zu}$ dieser Bewertung kommen Huston und Wright in ihrem Sammelreferat im Handbook of Child Psychology aus dem Jahr 1998, in dem sie auf Grundlage unterschiedlichster Studien (vom Experiment über Längsschnittstudien bis hin zur Metaanalyse) die Bedeutung der Massenmedien für die Entwicklung von Kindern und 
Jugendlichen zusammenfassen (vgl. Hoppe-Graff \& Kim, 2002). „Overall, the longitudinal studies support theories predicting that violence contributes to children's learned patterns of behavior or scripts in ways that can be manifested in behavior well beyond childhood." (Huston \& Wright, 1998, S. 1031). So wurde auch in einer auf über 17 Jahre angelegten Langzeituntersuchung der Columbia University bestätigt, dass exzessiver Fernsehkonsum vor allem bei den Jungen aggressives und antisoziales Verhalten in der Jugend und im Erwachsenenalter fördert (Johnson, Cohen, Smailes, Kasen \& Brook, 2002).

$\mathrm{Zu}$ einer ähnlichen Einschätzung gelangen auch Huesmann, Moise-Titus, Podolski und Eron (2003), die darüber berichten, dass unabhängig vom Geschlecht aggressives Verhalten im frühen Erwachsenenalter über den Konsum von Mediengewalt in der Kindheit vorhergesagt werden kann. In dieser Langzeitstudie wurde für eine Stichprobe von Kindern, die in den 1970ern und 1980ern aufwuchsen, festgestellt, dass der Konsum von gewalthaltigem Fernsehprogramm im Alter von 6 bis 10 Jahren mit aggressivem Verhalten ca. 15 Jahre später in Verbindung steht. Neben dem Konsum von Mediengewalt konnten auch die Identifikation mit dem gewalttätigen Charakter sowie der wahrgenommene Realismus der Gewaltszenen spätere Aggression vorhersagen. Die gefundenen Effekte blieben auch bei Kontrolle des sozioökonomischen Status, intellektueller Fähigkeiten und weiterer Variablen des elterlichen Erziehungsverhaltens bestehen.

„Präferenzen für Fernsehgewalt und die Neigung zur Ausübung von aggressivem Verhalten scheinen in einem bidirektionalen Zusammenhang zu stehen: Neben der Vorhersage der Aggressionsbereitschaft aus den früheren Sehgewohnheiten ist auch umgekehrt die Vorhersage der späteren Präferenz für gewaltsame Fernsehsendungen aufgrund der Gewaltneigung möglich." (Hoppe-Graff \& Kim, 2002, S. 913). ${ }^{11}$ Kriminologen gehen ferner davon aus, dass sich bei einer kleinen Risikogruppe von 5 bis 10 Prozent der männlichen Jugendlichen Gewaltinhalte unmittelbar auf ihre persönliche Gewaltbereitschaft auswirken. Bei diesen Jugendlichen, die aufgrund von familiären und sozialen Belastungsfaktoren (z. B. innerfamiliäre Gewalt, niedriges Selbstbewusstsein, emotionale Vernachlässigung oder Schulversagen) als besonders gefährdet einzustufen sind, können medial vermittelte Gewaltdarstellungen direkt als Identifikations- und Handlungsmuster fungieren (vgl. die Übersicht zum Stand der Erkenntnisse im Ersten Periodischen Sicherheitsbericht, 2001, S. 338-340). „Während das Ausmaß des allgemeinen Medienkonsums nur gering mit dem aggressiven, delinquenten und dissozialen Verhalten korrelierte, war dies beim Konsum gewalthaltiger Video-, Kino- und Fernsehfilme sehr deutlich der Fall. Die Bevorzugung von Freizeitaktivitäten und Medieninhalten, die Dispositionen zur Aggression und Delinquenz ausrichten und verfestigen können, steht im Rahmen eines

11 „Kunczik und Zipfel (1996) sprechen von einem sich selbst verstärkenden Prozess in dem Sinne, dass der Konsum von Mediengewalt die Wahrscheinlichkeit des Auftretens von aggressivem Verhalten, Einstellungen und Phantasien erhöht. Das wiederum steigert die Wahrscheinlichkeit, dass Gewalt in den Medien attraktiv ist und präferiert wird, wodurch sich die Zuwendung zu diesen Inhalten verstärkt" (Hoppe-Graff \& Kim, 2002, S. 913). 
allgemein devianteren Lebensstils. Dementsprechend fielen auch die Beziehungen des Problemverhaltens zum Substanzgebrauch (insbesondere Alkohol und illegale Drogen) sehr deutlich aus“" (Lösel \& Bliesener, 2003, S. 176).

Dass das Fernsehen einen gewaltsteigernden Effekt auf Vielseher haben kann, wird inzwischen als belegt angesehen (siehe oben). Fast einhellig wird jedoch die Vermutung geäußert, dass gewalthaltige Spiele eine noch stärker aggressionsfördernde Wirkung auf ihre Nutzer entfalten sollten als entsprechende Filme (für einen Überblick siehe: Kunczik \& Zipfel, 2004). So handelt es sich beim Spielen gewalthaltiger Spiele gegenüber der Rezeption von Filmen um einen aktiven Vorgang mit einer besonders hohen emotionalen Erlebnisintensität. Die Aufmerksamkeitsfokussierung ist weitaus höher und eine Identifikation bietet sich häufig nur mit der gewalttätig agierenden Spielfigur an. Gewalthandlungen des Spielers werden unmittelbar belohnt und haben in der Regel keine negativen, sondern positive Konsequenzen für die Erreichung der Spielziele. Somit vollzieht sich die Ausübung virtueller Gewalt über mehrere parallele Lernprozesse wie Modelllernen und Verstärkung, die wiederholt eingeübt werden. Gewalthaltige Spiele bieten zudem gegenüber Filmen eine sehr hohe Gewaltdichte und einen zunehmend größer werdenden Gewaltrealismus (Gentile et al., 2004; Kunczik \& Zipfel, 2004).

Gewaltelemente sind zudem ein gängiger, fast selbstverständlicher Bestandteil von Computerspielen. Kunczik und Zipfel (2004) zeigen in ihrem Überblick, dass die meisten und bestverkauften Computerspiele Gewalt enthalten und eine hohe Gewaltpräferenz bei den Konsumenten besteht. Dies spiegelt sich auch in Nutzerbefragungen wider. Einer Befragung von Siebt- und Achtklässlern zufolge $(n=367)$ haben gewalthaltige Spiele offenbar eine hohe Attraktivität für die Spieler, was sich darin zeigt, dass etwa die Hälfte der genutzten Lieblingsspiele Gewaltinhalte aufweist (Funk, 1993). Aus einer Befragung von Anderson und Dill (2000) an 227 Collegestudenten geht hervor, dass die von den Nutzern gespielten Spiele zu etwa 20 Prozent gewalthaltig sind. In einer Befragung von Gentile et al. (2004) geben nur 1 Prozent der Jungen und 16 Prozent der Mädchen $(n=670$, Acht- und Neuntklässler) an, Spiele ohne Gewalt zu bevorzugen. Jungen favorisieren also gewalthaltige Spielinhalte stärker als Mädchen. Gleichzeitig reglementieren Eltern die Nutzung von Gewaltspielen nur selten (Gentile et al., 2004).

In einer Untersuchung von Möller (2006) wurde der Zusammenhang von Gewaltspielkonsum und Aggression erstmals im Längsschnitt (6 Monate) analysiert, um Kausalaussagen zwischen beiden Phänomenen zu ermöglichen. Die Stichprobe bestand aus 349 Schülerinnen und Schülern siebter und achter Berliner Gesamtschulklassen (12-16 Jahre, $M W=13,3$ Jahre). Im Längsschnitt lagen noch 236 Datensätze zu beiden Messzeitpunkten vor. Erfasst wurden Gewaltmediennutzung und Gewaltmedienpräferenzen, Aggressivität als Persönlichkeitseigenschaft, normative Überzeugungen hinsichtlich aggressiven Verhaltens sowie feindselige Attributionsstile (Zuschreibungen). Im Längsschnitt zeigte sich hier wie der gewalthaltige Computerspielkonsum sich ein halbes Jahr später ereignende physische Aggressivität vorhersagen konnte. Bei Kontrolle der physischen Aggression beim ersten Messzeitpunkt 
zeigt sich bei den Jungen eine Wechselwirkung; wer aggressiv eingestellt ist, bevorzugt Gewaltspiele und wird dadurch in seiner Gewaltorientierung bestärkt. Bei den Mädchen bestand sogar eine einseitige Wirkrichtung von den Spielen auf physische Aggressivität. Die Längsschnittsdaten für den vergleichsweise kurzen Untersuchungszeitraum bestätigen wiederum die Annahme eines stärkeren Einflusses der Gewaltspielnutzung auf die physische Aggressivität als den umgekehrten Wirkzusammenhang. Zudem wurde festgestellt, dass die Wirkung auf Aggressivität über unterschiedliche Variablen vermittelt wird. Bei den Mädchen spielen offenbar insbesondere eine Veränderung normativer Überzeugungen und feindlicher Attributionstendenzen eine entscheidende Rolle, bei den Jungen eher die Akzeptanz physisch-aggressiver Handlungen. „Dass sich eine Beeinflussung der Akzeptanz aggressiven Verhaltens durch die Gewaltspiele dennoch und trotz Beachtung aller Modellpfade empirisch nachweisen ließ, zeigt die praktische Bedeutsamkeit dieser einzelnen Medienkonsumkomponente als eine Ursache der Ausbildung und Aufrechterhaltung aggressionsbegünstigender Einstellungen und Wissensstrukturen.“ (Möller, 2006, S. 198). Die Befunde interpretiert die Autorin insbesondere im Sinne des Modelllernens, indem die beobachteten Handlungen in das eigene Verhaltensrepertoire übernommen werden.

Die aggressionssteigernde Wirkung gewalthaltiger Computerspiele wird inzwischen kaum noch bezweifelt und auch ein kausaler Zusammenhang gilt für dieses Medium als gesichert (Emes, 1997). So stellen etwa Anderson und Dill (2000) fest, dass das Spielen von gewalthaltigen Spielen positiv mit aggressiver Delinquenz $(r=.46)$, mit nichtaggressiver Delinquenz $(r=.31)$ sowie mit einer aggressiven Persönlichkeit $(r=.22)$ korreliert. In einer metaanalytischen Auswertung von insgesamt 35 Forschungsberichten mit 54 voneinander unabhängigen Stichproben bestätigen Anderson und Bushman (2001) als Auswirkungen gewalthaltiger Spiele gesteigertes aggressives Verhalten $(r=.19)$, vermindertes prosoziales Verhalten $(r=-.16)$, gesteigerte aggressive Kognitionen $(r=.27)$, gesteigerte aggressive Affekte $(r=.18)$ und gesteigerte physiologische Erregung $(r=.22)$. In dieser Metaanalyse wurden nicht nur methodisch unterschiedliche Studien (Längsschnitt, Querschnitt, Experiment) berücksichtigt, sondern auch vielfältige Operationalisierungen der einzelnen Konstrukte.

Aggressives Verhalten wurde in den unterschiedlichen Studien z. B. operationalisiert über den Bestrafungslevel im Noise-Blasting-Paradigm ${ }^{12}$, Schlagen/Treten/Faustschlag/Beißen..., Kämpfe in der Schule, körperliche Angriffe (Lehrer, Freunde, Eltern), Raub, verbale Aggression, Fremdbeurteilung der Aggression (Lehrer, Freunde, Eltern), Selbstbeurteilung der Aggression, jugendliche Delinquenz und relationale Aggression (vgl. Anderson, C. A., Gentile \& Buckley, 2007, S. 39).

12 Versuchspersonen wird innerhalb eines fingierten Bestrafungsszenarios die Möglichkeit offeriert, andere Versuchspersonen nach Bedarf mit einem über Kopfhörer vermittelten lauten und unangenehmen Tonsignal zu bestrafen. Die Intensität des Signals kann dabei von den Versuchspersonen frei gewählt werden und als Maß für ihre aktuelle Aggressivität genutzt werden. 
In einer aktualisierten und differenzierteren Auswertung dieser metaanalytischen Daten verglichen die Autoren methodisch „einwandfreie“ Studien mit solchen, die in mindestens einem relevanten Merkmal Mängel aufwiesen (Anderson, C. A., 2004; Anderson, C. A. et al., 2007). Dabei zeigte sich, dass in den methodisch unproblematischen Studien über alle fünf aggressionsbezogenen Konstrukte hinweg deutlich größere Effektgrößen zu beobachten waren als in solchen Studien mit methodischen Mängeln ${ }^{13}$ : Gesteigertes aggressives Verhalten (unproblematisch: $r=.27$, methodische Mängel: $r=.16$ ), vermindertes prosoziales Verhalten $(r=-.27, r=-.12)$, gesteigerte aggressive Kognitionen $(r=.27, r=.20)$, gesteigerte aggressive Affekte $(r=.19, r=.09)$ und gesteigerte physiologische Erregung $(r=.22, r=.11)$. Bei der Berücksichtigung methodischer Mängel sind somit im Vergleich zur Metaanalyse aus dem Jahr 2001 vor allem bei den beiden Verhaltenskonstrukten, aggressives und prosoziales Verhalten, deutlich höhere Werte zu konstatieren.

Den Wirkprozess medialer Gewalt auf Aggressivität beschreiben Bushman und Anderson (2002) durch ihr General Aggression Model, das Konzepte anderer Theorien wie z. B. der kognitiven Lerntheorie, der Skript-Theorie oder des ExcitationTransfer-Modells integriert (vgl. Kunczik \& Zipfel, 2004). ${ }^{14}$ Unmittelbar während der Gewaltrezeption kommt es über die Prägung aggressiver Kognitionen, einem erhöhten Erregungszustand und der Auslösung aggressiver Gefühle wie Wut zu einem aggressionsähnlichen Zustand. Wiederholen sich diese Rezeptionsphasen nun über einen längeren Zeitraum, führen Lernprozesse und eine Verstärkung aggressionsbezogener Wissensstrukturen schließlich zu der Ausbildung einer aggressiven Persönlichkeit, indem aggressive Überzeugungen, Einstellungen, Wahrnehmungsschemata, Erwartungshaltungen und Verhaltensskripte verstärkt werden sowie gleichzeitig die Sensibilität für Aggressivität vermindert wird (Bushman \& Anderson, 2002; Gentile et al., 2004).

13 Beispiele für methodische Mängel sind: 1. Die nicht-gewalthaltige Computerspielbedingung enthielt Gewalt und war deshalb nicht als Kontrollbedingung geeignet. 2. Die gewalthaltige Computerspielbedingung enthielt nur ein geringes Maß oder keine Gewalt. 3. Die gewalthaltigen und nicht-gewalthaltigen Computerspielbedingungen unterscheiden sich so deutlich voneinander, dass das Ergebnis beeinflusst wird, wie z. B. darin, dass die nicht-gewalthaltige Bedingung schwieriger, langweiliger oder frustrierender ist als die gewalthaltige Bedingung. 4. Es wurde ein Vorher-Nachher-Design verwendet, aber nur der Durchschnitt aus Vorherund Nachhermessung wurde berichtet....(vgl. Anderson, C. A. et al., 2007, S. 165)

14 „Dem „General Aggression Model“ (GAM) liegt die Annahme zugrunde, dass die Ausübung von Gewalt v. a. auf dem Lernen, der Aktivierung und der Anwendung aggressionsbezogener, im Gedächtnis gespeicherter Wissensstrukturen basiert. Die drei Hauptkomponenten des Modells sind Input-Variablen (,Person“ und „Situation“), der gegenwärtige innere Zustand des Individuums und aus verschiedenen Einschätzungs- und Entscheidungsprozessen resultierende Ergebnisse.“ (Kunczik \& Zipfel, 2004, S. 111). 
Für die Forschergruppe um Funk ist unter den diskutierten Wirkfaktoren insbesondere die Desensibilisierung von entscheidender Bedeutung (Funk, Baldacci, Pasold \& Baumgardner, 2004), die nach dem General Aggression Model (GAM) von Bushman und Anderson (2002) zu den langfristigen Wirkmechanismen des Gewaltkonsums gehört. Eine Desensibilisierung für mediale Gewalt lässt sich beschreiben als eine allmähliche Abschwächung bzw. Löschung kognitiver, emotionaler und verhaltensbezogener Reaktionen auf die in Computerspielen spielerisch vermittelten Gewalthandlungen. Dieser Theorie zufolge würde also z. B. der immer wiederkehrende Anblick von gegnerischen Spielfiguren, die in Computerspielen verprügelt oder mit Kopfschüssen niedergestreckt werden müssen, Szenen, die bei ihrem ersten Auftreten möglicherweise noch Angst- oder Abwehrreaktionen hervorgerufen, vom Spieler zunehmend als weniger unangenehm erlebt. In der Folge kann sich der Spieler den Szenen immer problemloser zuwenden und damit die gewalthaltigen Spiele immer besser beherrschen, verändert in der Folge jedoch auch seine Wahrnehmungen und Bewertungen dieser Situationen. Aggression und Gewalt erscheinen in Folge zunehmend als legitimes und für die Zielerreichung wirksames Mittel. Die Gewöhnung für Computerspielgewalt geht mit einer Verminderung des Mitleides für Opfer realer Gewalt einher (Empathieverlust). ${ }^{15}$ Die eigene Hemmschwelle, Aggressionen offen auszuleben sinkt weiter ab (vgl. Möller, 2006).

Um diese Annahmen und einen eventuellen Transfer von Gewalterleben in Computerspielen auf normative Einstellungen in der Realität untersuchen zu können, entwarf die Autorengruppe um Funk (2004) eine Empathie-Messskala, den Childrens Empathy Questionnaire (CEQ). Beispielitems dieser Skala sind „If two kids are fighting, someone should stop them“ oder „When I see a kid who is upset it really bothers me“. Insgesamt untersuchten Funk et al. eine Stichprobe von 150 Grundschulkindern im Alter von durchschnittlich 10 Jahren. Zusätzlich wurden die Kinder zu ihren realen Gewalterfahrungen (KID-SAVE: KID-screen for adolescent violence exposure), ihrer Gewaltmediennutzung (Bildschirmspiele, Fernsehen, Kino und Internet) und ihren allgemeinen Einstellungen zu Gewalt befragt. Eine Regressionsanalyse unter Kontrolle des Geschlechts der Kinder ergab zunächst erwartungskonform, dass eine häufige Nutzung medialer Gewaltdarstellungen mit stärkerer Zustimmung zu gewaltbezogenen Einstellungen einhergeht. Hierbei entfalten sowohl Filme $(\beta=0.28, p<.001)$ als auch Computerspiele $(\beta=0.23, p<.001)$ eine steigernde Wirkung. Das besondere Ergebnis jedoch: Nur die Nutzung gewalthaltiger Computerspiele $(\beta=0.19, p<0.05)$ sagt geringere Werte auf der Empathieskala vorher; Gewalt aus Fernsehen, Kino und Internet hat hingegen keinen Einfluss auf die Empathiewerte der Kinder (Funk et al., 2004). Die Autoren diskutieren diesen und stellvertretend zu erleben und setzt sich aus der kognitiven Komponente der Perspektivenübernahme und der affektiven Komponente des Mitfühlens zusammen" (Möller, 2006, S. 125). 
Befund als Ausdruck der hochgradigen Aktivierung bei Computerspielen, in denen Kinder aktiv die Rolle des violenten Charakters einnehmen.

Die Selbstwahrnehmung von Spielern steht offenbar Forschungsdaten zur Desensibilisierungswirkung von Computerspielen diametral entgegen. In einer aktuellen explorativen Studie des British Board of Film Classification (Cragg, Taylor \& Toombs, 2007) wurden unter Verwendung qualitativer Methoden neben Eltern, Spielproduzenten, Spieldesignern und Redakteuren von Spielmagazinen auch Spieler zu ihrem subjektiven Erleben und ihren Meinungen zur Wirkung von Computerspielen befragt. Insgesamt fanden neben Gruppendiskussionen und Einzelinterviews 16 gepaarte Interviews mit Gelegenheits-, Normal- und Vielspielern (Alter 7 - 40 Jahre) unter anderem zu folgenden Fragekomplexen statt:

- Was glauben Spieler, welche Folgen die Nutzung von Computerspielen auf ihr Verhalten hat?

- Was glauben Spieler, welche Bedeutung der Interaktivität zukommt?

- Was denken Spieler über die Gewalt in Spielen?

- Wie wählen Spieler ihre Spiele aus und wie denken ihre Eltern darüber?

Natürlich zielen die Autoren dieser Befragungsstudie nicht auf Aussagen über die Wirkung von Computerspielen bzw. die differentielle Wirkung von Filmmedien vs. interaktiven Medien ab. Vielmehr sollen mit der Studie Meinungen und subjektive Einschätzungen verschiedener beteiligten Gruppen erfasst und beschrieben werden. Dies wird auch gleich zu Beginn von den Autoren unterstrichen:

„It was explicitly stated at the outset that the research could not resolve the issue of whether playing games desensitises people to violence in a way, or to a degree, that has regrettable effects on their behaviour. This important question was acknowledged to be beyond what the proposed research design would be able to deliver" (Cragg et al., 2007, S. 5)

Wenngleich Wirkungsaussagen innerhalb des Studiendesigns also nicht intendiert sind, wirft die Studie doch ein aufschlussreiches Licht auf die subjektive Selbstwahrnehmung der erfahrenen Spieler. Diese brachten in den Interviews zum Ausdruck, dass sie sich nicht in erster Linie für die Gewalt in den Spielen interessieren und sich wegen der Gewaltdarstellungen auch insgesamt keine Sorgen machten, weil sie die Grafik als weniger realistisch als die von Filmen und sich selbst als weniger emotional eingebunden empfinden. Eine hohe Konzentration bzw. der starke Wunsch, das Spiel zu gewinnen, sei zwar vorhanden, dies sei aber nicht mit einer starken emotionalen Beteiligung gleichzusetzen, so die Spieler. Insgesamt schätzen die Spieler die Gewalt in Filmen als weitaus schlimmer ein als die Gewalt in Computerspielen. Darstellungen in Filmen seien wirklichkeitsnäher als die Computergrafik, Filme erzeugten fesselndere Illusionen und die Spieler glauben, dass diese beiden Faktoren insgesamt ausschlaggebender seien als die Interaktivität des Computerspiels. Den jüngeren befragten Computerspielnutzern gefiel die Gewalt hingegen häufig nicht, sie fühlten sich unbehaglich, sich damit beschäftigen zu müssen. Einige jüngere Spieler ängstigen sich sogar vor Gewaltdarstellungen in Computerspielen und sie berichten, schlecht davon geträumt zu haben. 
Derartige Selbstberichte von Computerspielern unterschiedlicher Altersstufen sind typisch und stehen in Einklang mit der einschlägigen Wirkungsforschung zur desensibilisierenden Wirkung interaktiver Mediengewalt. Selbstverständlich kann nicht angenommen werden, dass ein Spieler die evtl. subtil einsetzende und sich schleichend vollziehende Desensibilisierung für Computerspielgewalt an sich selbst bemerken würde und kritisch reflektieren könnte. Im Gegenteil: In der Regel wird sich der routinierte Spieler einfach als besonders ,robust“ im Umgang mit Gewaltdarstellungen, insbesondere mit solchen aus Computerspielen, beschreiben und das Gefühl haben, die Gewalt habe weder eine besondere Wirkung auf ihn, noch belaste sie ihn besonders stark, ähnlich wie die befragten Spieler der britischen Nutzerbefragung. Dass Computerspielgewalt jedoch eine sehr mächtige und abschreckende Wirkung haben kann, bringen gleichfalls die kindlichen Spieler der britischen Nutzerbefragung zum Ausdruck, die über starke Abneigungen, Ängste und Albträume in Konfrontation mit der Computerspielgewalt berichten. Jüngere Nutzer sind interaktiver Gewalt in der Regel erst einem verhältnismäßig kurzen Zeitraum und vermutlich auch in weit geringerer Dosis ausgesetzt als ältere Nutzer. Aus diesem Grunde fallen Konfrontationen mit Gewaltdarstellungen bei jüngeren Kindern häufig noch weitaus emotionaler aus. Solche Berichte könnten also durchaus Anzeichen für eine sich bereits vollzogene Desensibilisierung für interaktive Gewaltdarstellungen sein, wenngleich sich diese Prozesse nur in Längsschnitt- oder experimentellen Designs zweifelsfrei nachweisen lassen. Diese Daten dürfen jedoch keinesfalls dahingehend interpretiert werden, dass Spiele schwächer wirken als Filme, nur weil dies der Selbstwahrnehmung der Nutzer entspricht. Derartige Aussagen sind wissenschaftlich nicht haltbar.

Bartholow, Bushman und Sestir (2006) konnten nun kürzlich nicht nur den physiologischen Nachweis einer Desensibilisierung durch gewalthaltige Bildschirmspiele erbringen, sondern ebenfalls aufzeigen, dass diese Desensibilisierung gleichzeitig unmittelbar mit einem aggressiveren Verhalten korreliert. 39 Probanden (Durchschnittsalter 19,5 Jahre) wurden zunächst im Rahmen eines quasiexperimentellen Versuchsdesigns zu ihrer Gewaltspielnutzung befragt und aus den Angaben wurde ein Gewaltexpositionsindex errechnet. Die Probanden wurden mit neutralen (z. B. Bild eines Fahrradfahrers), violenten (z. B. Mann bedroht Frau mit Waffe) und negativen (z. B. Baby mit Tumor im Gesicht) Bildern konfrontiert. Gleichzeitig wurden ihre EEG Reaktionen auf die Bilder abgeleitet und ereigniskorrelierte Potentiale der P300-Reaktion ${ }^{16}$ ermittelt. Das überraschende Ergebnis: Die Probanden mit einem hohen Gewaltexpositionsindex reagierten zwar genauso stark und schnell auf die negativen Bilder, reagierten jedoch deutlich schwächer und langsamer auf die Gewaltbilder als die Probanden mit geringer Gewaltexposition. Der Zusammenhang bleibt auch stabil, wenn die aggressiven Persönlichkeitsanteile der Probanden dabei kontrolliert werden. Geringere Reaktionen auf die Gewaltbilder, wie sie vornehmlich bei den Gewaltspielern auftraten, standen wiederum mit signifikant höheren

Die P300 entspricht einem elektrophysiologischen EEG-Korrelat einer Erkennungsreaktion eines seltenen Reizes, akustisch oder visuell, in einer Umgebung von häufigen Reizen. 
Werten in einem verhaltensnahen Aggressionstest in Beziehung. In diesem Verfahren mussten die Probanden, im Glauben gegen einen menschlichen Gegner anzutreten, möglichst schnell auf einen Hinweiston reagieren und konnten bei Erfolg ihren vermeintlichen Gegner mit einem lauten, unangenehmen Geräusch bestrafen. Sowohl Probanden mit geringeren corticalen Gewaltbilderreaktionen (Desensibilisierung) als auch Gewaltspielnutzer bestraften ihren Gegner mit lauteren Tonsignalen.

Damit konnte in dieser Studie erstmalig aufgezeigt werden, dass sich der Zusammenhang zwischen erhöhter Gewaltspielexposition und verhaltensbezogener Aggressionsmaße gleichzeitig auch über physiologische Maße im Sinne einer LangzeitDesensibilisierung auf Gewaltdarstellungen abbilden lässt. Weitere Analysen der Autoren zeigen auf, dass hauptsächlich die Gewaltspielnutzung, nicht die Spielzeit an sich von Bedeutung ist. Wenngleich eine größere Stichprobe in der Untersuchung sicherlich wünschenswert gewesen wäre, weist die Operationalisierung aufgrund des eingesetzten sehr verhaltensnahen Aggressionsparadigmas, der kontrollierten Variablen und der physiologischen Messung eine hohe Güte auf. Die Befunde zeigen zudem erstmalig auf, dass die durch Gewaltspiele hervorgerufene Desensibilisierung auch hinsichtlich sehr alltagsnaher Gewaltszenen (real-life-violence) und nicht etwa ausschließlich bei Bildern aus Gewaltspielen wirksam wird. Dies deutet darauf hin, dass Computerspiele nicht nur eine medienimmanente Desensibilisierung bewirken können, sondern die Desensibilisierung auch für emotionale Reaktionen auf reale Gewaltszenen von Bedeutung ist.

Physiologische Desensibilisierungseffekte konnten nicht nur für habituelle Gewaltspielnutzer sondern im Rahmen einer experimentellen Untersuchung auch für unmittelbare Gewaltspielexposition nachgewiesen werden. In dieser Untersuchung von Carnagey, Anderson und Bushman (2006) nahmen insgesamt 257 Versuchspersonen teil. Zunächst wurden als physiologische Maße Herzfrequenz (HR: HeartRate) und Hautwiderstand (GSR: galvanic skin response) im Rahmen einer Basislevelmessung erhoben. Zusätzlich wurden Fragebögen zu Aggressivität und Gewaltspielnutzung eingesetzt. Anschließend spielten die Probanden für 20 Minuten entweder ein gewalthaltiges oder ein gewaltneutrales Computerspiel. Zur Generalisierung der Ergebnisse wurde dabei jeweils aus einem Pool von vier unterschiedlichen Spielen ausgewählt. Nach der Treatmentphase folgten wieder Herzfrequenz- und Hautwiderstandsmessung, woraufhin die Probanden ein 10-minütiges Video zu sehen bekamen, in welchem nonfiktionale Gewalt (real-life-violence) aus vier unterschiedlichen Alltagskontexten gezeigt wurde. Währenddessen wurden wiederum kontinuierlich die physiologischen Daten aufgezeichnet. Die Probanden bewerteten zum Abschluss die Computerspiele hinsichtlich verschiedener 10-stufiger Einschätzungsskalen (z. B. Unterhaltungserleben und Gewaltempfinden).

Die Ergebnisse zeigen auf, dass die Herzfrequenz der Probanden, die für $20 \mathrm{Mi}-$ nuten ein gewalthaltiges Spiel gespielt hatten, während des Anblicks echter Gewalt auf dem gleichen moderaten Level verblieb, während bei den Probanden der NichtGewalt-Bedingung eine Erhöhung der Herzfrequenz zu verzeichnen war $(F=4,86$, $p<0.05$ ). Die vorausgegangene Gewaltspielnutzung machte die Spieler also offenbar immun gegen eine Erregungserhöhung aufgrund echter Gewalt, was die Autoren 
als physiologisches Anzeichen einer Desensibilisierung interpretieren. In der Hautwiderstandsmessung kam während des Anblicks realer Gewalt sogar eine Erregungsverminderung der Gewaltspieler gegenüber der Spieler neutraler Games zum Ausdruck. Deren Erregungslevel blieb hingegen wiederum konstant $(F=4,67$, $p<0.05)$.

Die Befunde zeigen deutlich, dass schon das 20-minütige Spielen eines gewalthaltigen Computerspiels unmittelbar physiologische Erregungen beim Anblick tatsächlicher Gewalt reduzieren kann. Zudem zeigen die Daten, dass die Befunde robust in vielfältigen Unterpopulationen auftreten. Weder das Geschlecht der Probanden noch die Ausprägung aggressiver Persönlichkeitsanteile oder die vorliegende Präferenz für Computerspiele zeigte einen Einfluss auf die festgestellte Desensibilisierungswirkung. Die Autoren sehen daher sogar die gesellschaftliche Gefahr einer global zunehmenden und unbemerkten Desensibilisierung durch Gewaltmedien gegeben: „Older children consume increasingly threatening and realistic violence, but the increases are gradual and always in a way that is fun. In short, the modern entertainment medial landscape could accurately be described as an effective systematic violence desensitization tool." (Carnagey et al., 2006, S. 7).

\section{Moderierende Einflussfaktoren}

Welche Wirkungen weitere Variablen entfalten können wie etwa das Alter, das Geschlecht oder das soziale Umfeld, lässt sich Untersuchungen entnehmen, die sich mit der differentiellen Wirkung von Mediengewalt auf unterschiedliche Rezipienten beschäftigt haben (für einen Überblick über diese und weitere Einflussfaktoren siehe Kunczik \& Zipfel, 2004). Forschungsbefunde zum Zusammenhang zwischen dem Alter und der Wirkung medialer Gewaltdarstellung bestätigen die Vermutung, dass sich diese insbesondere auf jüngere Kinder stärker auswirkt (vgl. Paik \& Comstock, 1994). Begründet werden mögliche altersabhängige Unterschiede vor allem durch unterschiedlich weit entwickelte Fähigkeiten zur Informationsverarbeitung, wie z. B. dem Verständnis von Medieninhalten, der Realitäts-Fiktions-Unterscheidung, der Empathiefähigkeit, sowie dem schlussfolgernden Denken (vgl. Kunczik \& Zipfel, 2004). Von besonderer Bedeutung ist demnach die Wahrnehmung der dargestellten Gewaltdarstellungen und -inhalte. So zeigt eine britische Studie von Hargrave (2003) mit 9- bis 13-jährigen Kindern $(\mathrm{n}=83)$, dass Kinder Gewalt weniger nach deren Merkmalen als nach dem Grad ihrer emotionalen Wahrnehmung beschreiben, und diese häufig als furchteinflößend wahrnehmen (vgl. Kunczik \& Zipfel, 2004). Eine klare Linearität zwischen Gewaltwirkung und Lebensalter lässt sich jedoch nicht feststellen, was hauptsächlich durch die mangelnde Vergleichbarkeit des verwendeten Stimulusmaterials und das aus forschungsethischer Perspektive problematische Untersuchungsfeld bedingt ist.

Immer wieder wurde auch die Vermutung geäußert, dass das Geschlecht des Nutzers für die Wirkung von Gewalt von Bedeutung sein könnte. Wie wir bereits ausgeführt haben, nutzen Jungen Computerspiele häufiger als Mädchen und haben auch 
häufiger einen Fernseher im eigenen Zimmer (KFN Schülerbefragung 2005; Feierabend \& Klingler, 2003a, 2003b; Feierabend \& Rathgeb, 2006a, 2006b). Des Weiteren konnte festgestellt werden, dass Jungen bei Computerspielen gewalthaltige Spiele wie z. B. First-Person-Shooter deutlich stärker favorisieren als Mädchen (Anderson, C. A. \& Dill, 2000). Für Filme stellt sich dies ähnlich dar: Aus einer 10jährigen Längsschnittstudie mit ca. 4.500 Schülern folgern Fuchs, M. et al. (2005, S. 188): „Die Gruppe derer, die sich täglich Kriegs-, Horror- und/oder Sexfilme ansehen, wird immer größer und besteht 2004 fast nur noch aus männlichen Schülern“. Es lässt sich somit eine stärkere mediale Gewaltexposition männlicher Nutzer feststellen. Damit sind Jungen insgesamt stärker von den schädlichen Auswirkungen medialer Gewalt betroffen.

Darüber hinaus ist jedoch von Interesse, ob Mädchen und Jungen auf ähnliche Weise auf mediale Gewaltdarstellungen reagieren, oder ob der Einfluss geschlechtsspezifische Differenzen aufweist. Hier herrscht eine uneinheitliche Befundlage. In experimentellen Studiendesigns hat sich gezeigt, dass gewalthaltige Filme einen stärkeren Einfluss auf Jungen haben, als auf Mädchen. Dies konnte jedoch in nichtexperimentellen Designs nicht bestätigt werden (vgl. Paik \& Comstock, 1994). So zeigt eine neuere Metaanalyse von Bushman und Anderson (2002) auch für Computerspiele, dass sich mediale Gewaltdarstellungen auf beide Geschlechter ähnlich auswirken und sich keine nennenswerten geschlechtsspezifischen Wirkungsmuster ergeben.

Dem sozialen Umfeld von Kindern und Jugendlichen wird nach aktueller Befundlage vor allem eine Moderatorfunktion zwischen Medienkonsum und Entstehung bzw. Ausübung von Gewalthandeln zugesprochen. Der elterliche Fernsehkonsum sowie deren allgemeiner Erziehungsstil (vgl. Weiler, 1999), die Peer-Group und ihre normative Orientierung (vgl. Fuchs, M. et al., 2001; Fuchs, M. et al., 2005) und nicht zuletzt das schulische Umfeld (Döbler, Stark \& Schenk, 1999) können sich dabei sowohl auf den Medienkonsum als auch auf die Ausübung von Gewalt auswirken. Eine Berücksichtigung dieser Faktoren in Studien zur Medienwirkung ist daher unerlässlich.

\subsubsection{Mediengewalt, Aggressivität und die Bedeutung für den Schulerfolg}

Mittels seiner negativen Wirkung auf Aggressivität und aggressives Verhalten kann sich der Konsum gewalthaltiger Medieninhalte auch negativ auf Schulleistungen auswirken. Nach Anderson, D.R. et al. (2001) kann Aggression zu einer Spirale von weniger Leistung, geringerer Arbeitsqualität, schlechteren Noten, verringerter Erfolgsmotivation und somit zu einem negativen Kreislauf von Schulversagen, einer Entfremdung von der Kultur des Erfolges und den Zielen, die durch die Schule repräsentiert werden, führen (vgl. MacBeth, 1996; Williams, 1986). Es gibt zahlreiche Belege dafür, dass frühe aggressive Verhaltensmuster mit geringerem nachfolgendem Schulerfolg korrelieren (z. B. Anderson, D. R. et al., 2001; Bates, Pettit \& Dodge, 1995; Kokko \& Pulkkinen, 2000). Kokko und Pulkinnen (2000) konnten 
zum Beispiel Zusammenhänge zwischen aggressivem Verhalten in der Kindheit und späterer Langzeitarbeitslosigkeit im Erwachsenenalter aufzeigen. Die ca. 300 Teilnehmer dieser Langzeitstudie waren Teil der ,Jyväskylä Longitudinal Study of Personality and Social Development", welche die zu Beginn 8-jährigen Versuchspersonen bis ins Alter von 36 Jahren begleitete. Daten wurden im Alter von 8, 14, 27 und 36 Jahren erhoben. Als ein Ergebnis zeigte sich, dass das durch die Lehrkräfte eingeschätzte aggressive Verhalten ${ }^{17}$ im Alter von 8 Jahren über Prozesse der Fehlanpassung mit späterer Langzeitarbeitslosigkeit ${ }^{18}$ korrelierte. So konnte aggressives Verhalten im Alter von 8 Jahren schulische Fehlanpassung ${ }^{19}$ im Alter von 14 Jahren $(r=.53)$ vorhersagen, welche direkt $(r=.31)$ und indirekt (vermittelt über „Problemtrinken“ ( $r=.49)$ und Mangel an Beschäftigungsalternativen $(r=.43))$ mit Langzeitarbeitslosigkeit verbunden war. Des Weiteren hing schulische Fehlanpassung unmittelbar mit weniger Erfolg in der Schule, d. h. schlechteren Noten $(r=.84)$, einem mangelndem Interesse an Schularbeiten $(r=.79)$, mehr Bestrafungen in der Schule $(r=.76)$ und mehr Schuleschwänzen $(r=.69)$ zusammen.

In anderen Studien konnte der Zusammenhang zwischen dem häufigen Konsum gewalthaltiger Fernsehprogramme und schlechteren Schulleistungen direkt aufgezeigt werden (vgl. Huesmann \& Eron, 1986). So berichten Huesmann und Eron (1986) in ihrer Untersuchung $(n=758)$ über negative Zusammenhänge zwischen Schulerfolg, gemessen mit dem California Achievement Test, und aggressivem Verhalten des Kindes (Jungen: $r=-.40$, Mädchen: $r=-.38$, jeweils $p<.001$ ), sowie dem Konsum gewalthaltiger Fernsehprogramme (Jungen: $r=-.25, p<.01$, Mädchen: $r=-.11$, ns). Je geringer der Erfolg in der Schule, desto mehr aggressives Verhalten zeigte das Kind, desto mehr gewalthaltige Fernsehprogramme wurden konsumiert, und desto mehr glaubte das Kind, dass die gezeigte Fernsehgewalt dem wirklichen Leben entspricht. „Low achievement may be a frustrator, instigating aggressive responses, and a marker of a reduced capacity to deal intelligently with difficult situations. The low achieving child may turn to television violence to obtain vicariously the success that he or she cannot obtain in school. In turn, increased television viewing and the aggression it spawns may contribute to a further decline in academic achievement." (Huesmann \& Eron, 1986, S. 67). Die bedeutsame Korrelation kann somit entweder bedeuten, dass das Betrachten gewalthaltiger Fernsehprogramme den Schulerfolg beeinflusst oder, dass Schüler mit schlechteren Noten eher ein gewalthaltiges Fernsehprogramm auswählen (Anderson, D. R. et al., 2001). Eine Längsschnittstudie derselben Forschergruppe, in der sowohl der sozioökonomische Status als auch das Bildungsniveau der Eltern kontrolliert wurden, gab jedoch Hin-

17 Aggressives Verhalten wurde von den Lehrkräften mit folgenden vier Items beurteilt: ,verletzt andere Kinder, wenn wütend, z. B. durch schlagen, treten oder das Werfen von etwas“, „tritt Möbel oder andere Dinge, wenn wütend über etwas“, ,greift andere ohne Grund an“, ,ärgert kleinere oder schwächere Gleichaltrige, wenn wütend über etwas“).

18 Langzeitarbeitslosigkeit $=$ mehr als 24 Monate arbeitslos im Alter von 27 bis 36 Jahren.

19 Schulische Fehlanpassung wurde über vier Variablen operationalisiert: Erfolg in der Schule Noten, Interesse an Schularbeiten, Bestrafung in der Schule, Schuleschwänzen. 
weise darauf, dass ein verringerter Schulerfolg eher die Folge als die Ursache des Konsums gewalthaltiger Fernsehprogramme ist (vgl. Anderson, D. R. et al., 2001; Huesmann, Eron, Lefkowitz \& Walder, 1984).

Abschließend können für die Inhaltshypothese die folgenden Gesichtspunkte hervorgehoben werden: Die insbesondere von männlichen Kindern und Jugendlichen präferierten Unterhaltungsmedien zeichnen sich häufig durch einen hohen Anteil von Gewaltdarstellungen sowie eine starke inhaltliche Fokussierung auf Gewalthandlungen aus. Forschungsdaten weisen zudem darauf hin, dass der Anteil an medialer Gewalt weiterhin im Steigen begriffen ist und insbesondere der Gewaltrealismus in Computerspielen Jahr für Jahr aufgrund verbesserter Hardware zunimmt. Bezüglich der Wirkungen dieser Inhalte wird die Forschungslage zunehmend differenzierter.

Im Bereich der Filme konnte mit unterschiedlichster Methodik gezeigt werden, dass für Aggressivität und Gewalthandeln relevante Faktoren wie z. B. aggressionsbezogene Verhaltensskripte oder die Fähigkeit zum Mitleid mit Opfern gerade durch einen längerfristigen und regelmäßigen Gebrauch von Gewaltinhalten negativ beeinflusst werden. Wenngleich immer auch andere Variablen einen Einfluss auf diesen Zusammenhang nehmen können, gilt die grundsätzlich aggressionssteigernde Wirkung gewaltbezogener Medien als empirisch belegt. Die Besonderheiten des interaktiven Mediums Computerspiel weisen zudem darauf hin, dass hier ein besonders hohes Wirkpotential zu befürchten ist. Bei einer Zunahme von Aggressivität ist neben anderen Konsequenzen eine Verminderung schulischer Leistungen und im Extremfall ein zunehmender Ausstieg aus der Leistungsgesellschaft zu befürchten.

\subsection{Löschungshypothese}

Der dritte große Ansatz zur Erklärung des Zusammenhangs intensiven Mediennutzungsverhaltens und herabgesetzter Leistungsfähigkeit in der Schule ist die sog. Löschungshypothese. Sie stützt sich auf Erkenntnisse gedächtnispsychologischer und neurobiologischer Forschung, die darauf hinweisen, dass sowohl mediale Gewaltdarstellungen als auch die besonderen biophysiologischen Erregungsmuster, insbesondere bei der Nutzung von Computerspielen, einen unmittelbaren Einfluss auf Informationsverarbeitungsprozesse des Nutzers nehmen können.

Im Bereich der Computerspiele wurde der Einfluss des Spielens auf kognitive Prozesse beim Lernen bereits mehrfach empirisch belegt, jedoch oftmals verknüpft mit einseitigen Schlussfolgerungen auf das kompetenzförderliche Potential von Computerspielen. Computerspieler lernen tatsächlich, jedoch in erster Linie Spielinhalte und Kompetenzen, die für die Bedienung des jeweiligen Spiels erforderlich sind. So werden bei den sehr beliebten First-Person-Shootern insbesondere für den Spielerfolg relevante Elemente erlernt (z. B. Aufbau der Spielumgebung, Steuerbefehle, Gegnerbewegungen, Waffenfunktionen usw.). Dieses Lernen vollzieht sich ebenso genussvoll wie intensiv, denn charakteristisch für das Spielen von Compu- 
terspielen ist eine starke Dopaminausschüttung. So ermittelten Koepp et. al. (1998) eine um 100 Prozent erhöhte Ausschüttung von Dopamin beim Spielen von Gewaltspielen. Jeder Treffer, jeder „Frag“ belohnt den Spieler nicht nur unmittelbar durch Punkte, Upgrades oder das Voranschreiten in der Spielgeschichte, sondern auch durch die Freisetzung von Dopamin.

Die Autoren führen hierzu aus, dass gewalthaltige Computerspiele die erste reine Verhaltensbedingung seien, bei der eine bedeutsame Dopaminausschüttung festgestellt wurde. Dopamin als „Glücksbotenstoff“ gilt gleichzeitig als zentraler Modulator des Lernsystems, eine Dopaminausschüttung verstärkt aktuelle Lernvorgänge (vgl. Spitzer, 2003). So verwundert es nicht, dass langjährige Spieler zu wahren „Profis“ in den von ihnen favorisierten Spielen werden, immer effektiver in der Benutzung der Eingabemedien und immer schneller in ihren Reaktionen. Die Lernprozesse werden hier als weitgehend intensiver angesehen als bei passiver Medienrezeption in Filmen (Scheich, 2006). Spieler lernen die Beherrschung des Virtuellen also effektiv. Einige wenige Spieler schaffen es sogar in die „E-Sport“-Ligen und stellen als professionelle Computerspieler auf internationalen Turnieren ihr Können unter Beweis. Die Skill-Unterschiede zwischen einem Gelegenheitsspieler und einem professionellen Computerspieler sind erstaunlich und demonstrieren, dass nur jahrelange Lernprozesse diese Fähigkeiten in der Beherrschung des Spiels ermöglichen.

Die Berichte über erfolgreiche Computerspieler und ihre besonderen Fähigkeiten, die sie durch die Nutzung des Mediums haben erwerben können (siehe Anfang des Kapitels), werden bis heute immer wieder herangezogen, um die positiven Seiten der modernen Freizeitbeschäftigung „Computerspiel“ zu unterstreichen. Ob die zumeist zeitintensiven Lernerfahrungen jedoch auch in den lebenspraktischen Alltag übertragen werden können, ob also sinnvolle Transferprozesse stattfinden, wird kaum kritisch hinterfragt und auch nicht empirisch untersucht. So berichtet Horn in einem Interview für die Bundeszentrale für politische Bildung ernste Zweifel an solchen Transferprozessen: „Der Rückschluss vom Computerspiel unmittelbar auf die Realität und das Lernen für das richtige Leben ist mir zu einfach. Ich glaube, beim Computerspielen lernt man vor allen Dingen eines: nämlich besser Computerspielen.“(Horn, 2006).

Doch nicht nur die Alltagsrelevanz der in Computerspielen gemachten Lernerfahrungen kann stark angezweifelt werden, auch nichtmediale Lernprozesse können durch das Computerspielen unmittelbar in Mitleidenschaft gezogen werden. An dieser Stelle setzt die Löschungshypothese an. Sie besagt, dass durch stark emotionalisierendes Medienerleben eine Verdrängung paralleler bzw. konkurrierender Lernprozesse, wie sie zum Beispiel im Rahmen schulischen Lernens stattfinden, verursacht werden könnte. Dass emotionale Ereignisse unsere Wahrnehmung und unsere Erinnerungen gravierend beeinflussen, ist eine bekannte Alltagserfahrung. So können sich viele Menschen noch nach Jahren detailliert an den eigenen Tagesablauf während eines bedeutenden politischen Ereignisses erinnern. Gewaltopfer sind hingegen häufig nicht in der Lage, sich an begleitende Umstände wie z. B. vorausgegangene Ereignisse zu erinnern. Gleichzeitig ist wissenschaftlich bereits eingehend 
untersucht, dass der Anblick und das Miterleben emotionalisierender Bilder, insbesondere von Gewaltbildern, in aller Regel mit starken Gefühlszuständen verbunden sind. Die Rezeption emotional geladener Medieninhalte (z. B. aus Horrorfilmen oder gewalthaltigen Computerspielen) geht mit einer Erhöhung physiologischer Stressparameter einher wie Herzschlag, Blutdruck, Adrenalin, Cortisol und Noradrenalin (Baldaro et al., 2004; Carnagey et al., 2006; Griffiths \& Dancaster, 1995; Mazur, Susman \& Edelbrock, 1997; Skosnik, Chatterton, Swisher \& Park, 2000). Durch eine solche Aktivierung der Stressachse ${ }^{20}$ kann eine Beeinträchtigung vorangegangener Lernvorgänge ausgelöst werden (Cahill \& McGaugh, 1996; McGaugh, 2000). Die Gefahr einer solchen Lernbeeinträchtigung besteht umso stärker, je kürzer die Lernerfahrung zurückliegt, da über den Hippocampus vermittelte deklarative Gedächtnisinhalte noch nicht konsolidiert (gefestigt) sind (Izquierdo \& Medina, 1997).

Die Auswirkungen auf das Lernen sind jedoch immer eine Frage der Stressdosis: Ein geringes emotionales Erleben bzw. moderater Stress wirken durchaus anregend und können die Qualität vorangegangener Lernvorgänge sogar steigern (Cahill \& McGaugh, 1996; McGaugh, 2000). Erst ab einer gewissen Stärke wirkt sich die emotionale Erregung negativ auf zuvor akquirierte Gedächtnisinhalte aus. Die Frage ist also, ob brutale Filme und Gewaltspiele das adäquate, lernförderliche oder zumindest lernneutrale Erregungsmaß überschreiten. Das subjektive Empfinden ist hierbei oftmals irreführend. Die bereits erwähnte Untersuchung der Freiburger Forschergruppe um Myrtek zeigt am Beispiel des Fernsehens auf, dass der durch Medien hervorgerufene Stress oftmals unterschätzt und selten bewusst wahrgenommen wird (Myrtek, 2003; Myrtek \& Scharff, 2000). In dieser Studie wurden biophysiologische Stressparameter von Schülern anhand mobiler Messgeräte im Tagesverlauf erfasst und ausgewertet. Obwohl die Schüler angaben, in der Schule den meisten Stress empfunden zu haben, zeigen die Daten, dass gerade vor dem Fernseher der meiste Stress erlebt wurde. „Es lässt sich somit kein ,Schulstress', sondern ein „Fernsehstress' ausmachen“ (Myrtek, 2003, S. 458). Spitzer (2005) sieht in diesen Befunden eine besondere Gefahr auch für schulisches Lernen: „Wer morgens in der Schule döst und seine Pulsfrequenz nahe der Schlafgrenze wenig moduliert, der wird nichts lernen. Wer dann nachmittags Gewaltfilme oder Horrorvideos mit Pulsbeschleunigung betrachtet, der lernt die Gewalt besonders gut.“ (Spitzer, 2005, S. 132). Scheich (2006) sieht hier eine unmittelbare Gefahr gegeben, da durch die gesteigerte Mediennutzung andere Lernprozesse verdrängt werden könnten. So führt er aus, dass konkurrierende Mediengewohnheiten von Kindern und Jugendlichen die Gedächtniskonsolidierung von Erziehungs- und Bildungsinhalten beeinträchtigen und damit in Verdacht stehen, für den bereits nachgewiesenen negativen Zusammenhang von Mediennutzung und Schulleistung verantwortlich zu sein.

20 Zunächst Ausschüttung von Adrenalin und Noradrenalin (aus dem Nebennierenmark) vermittelt über eine Aktivierung des sympathischen Nervensystems. Langfristig kommt es vermittelt über den Hypophysenvorderlappen zu einer Ausschüttung von Glucocorticoiden in der Nebennierenrinde. 
„Es ist offensichtlich, dass Medienkonsum mit stark emotional wirksamen Inhalten eine bevorzugte Verankerung solcher Informationen bewirkt. Dies geschieht in Konkurrenz zu weniger aufwühlenden Erfahrungen z. B. im Schulalltag. (...) Die Verankerung einer Information im Langzeitgedächtnis dauert mehr als 24 Stunden. Wird während dieser Zeit die Information wiederholt oder durch ähnliche Informationen variiert, verstärkt sich die Verankerung im Langzeitgedächtnis. Dies erklärt einerseits den massiven Effekt von regelmäßigem Langzeitkonsum bestimmter Medien, andererseits aber auch den Sinn nachmittäglicher Hausaufgaben in der Schule oder Ganztagsschule mit Stoffvertiefungen am Nachmittag. Nimmt TV-Konsum oder Computerspielen die zeitlich und emotional dominierende Rolle im Tagesverlauf ein, hat Schulstoff keine Chance fest im Langzeitgedächtnis verankert zu werden." (Scheich, 2006, S. 227f)

Einige Studien aus der Werbewirkungs- und Nachrichtenforschung unterstreichen diese Annahme. So berichtet Bushman (1998) die Daten aus einer Serie von insgesamt drei Experimenten: Neutrale Werbebotschaften wurden jeweils in gewalthaltige oder in gewaltneutrale Filme eingebettet. Dabei zeigte sich bei studentischen Versuchspersonen $\left(\mathrm{n}_{1}=200, \mathrm{n}_{2}=200, \mathrm{n}_{3}=320\right)$ eine signifikant schlechtere Erinnerung an die Werbung, wenn diese in gewalthaltigen Filme platziert wurde. Dies galt gleichsam für verschiedene Dimensionen von Gedächtnisleistung: Freie Reproduktion der Markennamen, Wiedererkennung der Markennamen und Wiedererkennung der Werbebotschaft. Unter Kontrolle weiterer Störvariablen zeigte sich der Effekt unbeeinflusst von Arousal und positiven Affekten. Jedoch zeigte sich, dass insbesondere der durch die Gewaltfilme ausgelöste Ärger zu einer erhöhten Vergessensrate beiträgt. In einem weiteren Experiment an einer weniger selektiven Stichprobe $(\mathrm{n}=328)$ fanden sich diese Effekte auch noch verzögert nach 24 Stunden, und gleichsam bei Männern und Frauen, unterschiedlichen Altersklassen und unabhängig davon, ob Gewaltinhalte durch die Versuchspersonen präferiert wurden oder nicht (Bushman \& Bonacci, 2002).

Weitere Befunde lassen sich der Nachrichtenforschung entnehmen. Hier wurde festgestellt, dass solche Informationen, die innerhalb von Nachrichten mit Gewaltbildern präsentiert wurden, besser erinnert werden als solche, die innerhalb von Nachrichten ohne Gewaltbilder präsentiert wurden. Die Erinnerung an Informationen jedoch, die vor einem Nachrichtenbeitrag aufgenommen wurden, fällt schwächer aus, wenn dieser Nachrichtenbeitrag verstörende Gewaltbilder beinhaltet (Lang, Newhagen \& Reeves, 1996). Die Autoren interpretieren ihre Befunde im Sinne des „limited capacity information processing approach“ als Ausdruck eines Verarbeitungsdefizits. Um die augenblicklich erhöhte Verarbeitungsleistung während der Rezeption der Gewaltbilder zu ermöglichen, werden Ressourcen von anderen, noch unvollendeten Verarbeitungsvorgängen abgezogen (vgl. Lang et al., 1996).

In diesen Studien sind jedoch die Lerninhalte immer auch Medieninhalte, weshalb die Ergebnisse nicht direkt auf schulische Lerninhalte übertragen werden können. Welchen Einfluss gewaltmediale Darstellungen in Filmen und Computerspielen auf vorangegangene schulische Lernprozesse nehmen, wurde bislang nicht erforscht. Schulisches Lernen zeichnet sich allerdings durch Muster aus, die es in vielerlei Hinsicht störanfällig machen. Lernstoff wird wiederholt eingeübt, bis eine Festigung des Gelernten erfolgt ist. Jede Wiederholung des Lernstoffs führt insgesamt zu einer 
Fortsetzung der Konsolidierung, bringt die Informationen jedoch auch für einen gewissen Zeitraum in einen instabilen Zustand, der sehr sensibel auf emotionale Vorgänge reagiert.

Die gerade beim Spielen gewalthaltiger Spiele festgestellten hohen biophysiologischen Erregungsmuster geben hier Anlass zur Sorge. Hier könnten insbesondere subpopulationsspezifische Gefährdungen gegeben sein, indem sich Menschen mit bestimmten Persönlichkeitsmustern oder Biographien als besonders anfällig für eine lernschädigende Wirkung von Gewaltmedien erweisen. So ist beispielsweise bekannt, dass die Aktivierung durch Gewaltspiele besonders stark ausfällt, wenn der Rezipient aggressive Persönlichkeitszüge aufweist (Anderson, C. A. \& Dill, 2000). Weitere Beispiele lassen sich der Medienwirkungsforschung zu Mediengewalt und Aggressivität entnehmen, die differentielle Effekte erwarten lassen (vgl. Kapitel 3.2). Gleichfalls könnten die Quantität der Mediennutzung und die Qualität der präferierten Inhalte von entscheidender Bedeutung sein. Die Stärke der physiologischen Reaktion lässt sich demnach nicht pauschal angeben und variiert innerhalb des interindividuell spezifischen Rezeptionskontextes. Wie sehr demnach gewalthaltige Filme oder Spiele im Alltag von Schülerinnen und Schülern über kognitive Mechanismen für den negativen Zusammenhang zwischen Schulleistung und Mediennutzung verantwortlich sind, lässt sich zu diesem Zeitpunkt noch nicht abschätzen, da entsprechende Untersuchungen fehlen. Hierzu muss weitere Forschung abgewartet werden.

Nachdem im theoretischen Abschnitt dieses Textes unterschiedliche Wirkhypothesen zum Zusammenhang von Mediennutzung und Schulleistung vorgestellt und bewertet wurden, sollen im nachfolgenden Teil Befunde aus unserem laufenden Forschungsprojekt vorgestellt werden, die die bestehende Erkenntnislage um einige zentrale Aspekte bereichern. 